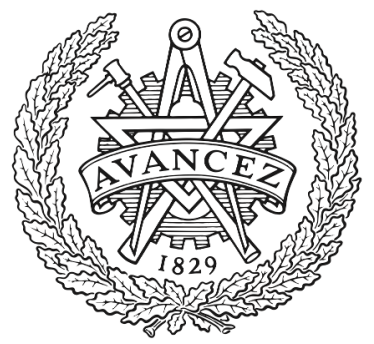

CHALMERS

UNIVERSITY OF TECHNOLOGY

\title{
A parametric study on the influence of boundary conditions on the longitudinal pressure gradient in CFD simulations of an automotive wind
}

Downloaded from: https://research.chalmers.se, 2023-04-26 12:21 UTC

Citation for the original published paper (version of record):

Ljungskog, E., Sebben, S., Broniewicz, A. et al (2017). A parametric study on the influence of boundary conditions on the longitudinal pressure gradient in CFD simulations of an automotive wind tunnel. Journal of Mechanical Science and Technology, 31(6): 2821-2827. http://dx.doi.org/10.1007/s12206-017-0525-2

N.B. When citing this work, cite the original published paper. 


\title{
A Parametric Study on the Influence from Boundary Conditions on the Longitudi-
}

\section{nal Pressure Gradient in CFD simulations of an Automotive Wind Tunnel ${ }^{\dagger}$}

\author{
Emil Ljungskog ${ }^{1 *}$, Simone Sebben ${ }^{1}$, Alexander Broniewicz ${ }^{2}$ and Christoffer Landström ${ }^{2}$ \\ ${ }^{1}$ Department of Applied Mechanics, Chalmers University of Technology, 41296 Gothenburg, Sweden \\ ${ }^{2}$ Volvo Cars, 40531 Gothenburg, Sweden
}

\begin{abstract}
Computational Fluid Dynamics (CFD) is an important and extensively used tool for aerodynamic development in the vehicle industry today. As manufacturers wish to substitute physical tests on prototype vehicles with virtual simulations, validation of the virtual methods by comparison to wind tunnel experiments is a must. A proper validation can only be performed if the wind tunnel geometry with representative boundary conditions is included in the numerical simulation and if the flow is well predicted for the empty wind tunnel. One of the important flow parameters to predict is the longitudinal pressure distribution in the test section, which is dependent on both the wind tunnel geometry and the settings of the boundary layer control systems. This work investigates the effects of inlet angularity and different boundary layer control systems, namely basic scoop suction, distributed suction and moving belts, on the longitudinal pressure distribution in the Volvo Cars full scale aerodynamic wind tunnel using CFD and a systematic design of experiments approach. The study shows that the different suction systems used to reduce boundary layer thickness upstream of the vehicle have statistically significant effects on the longitudinal pressure distribution in the test section. However, the estimated drag difference induced on a typical vehicle by the difference in horizontal buoyancy between the tested settings is within the test-to-test accuracy of the physical wind tunnel, leading to the conclusion that force calculations in simulations are fairly insensitive to the tested parameters on the intervals investigated.
\end{abstract}

Keywords: CFD; Design of Experiments; Automotive Wind Tunnel; Boundary Layer Control

\section{Introduction}

Computational Fluid Dynamics (CFD) methods for evaluation of the aerodynamic properties of road vehicles are valuable tools in the vehicle industry today, and are becoming even more important as manufacturers strive to substitute physical prototype testing with virtual methods in order to reduce lead time. It is therefore important to be able to validate the simulation results obtained from CFD, by for example comparison with wind tunnel experiments. Traditionally, virtual aerodynamic simulations of road vehicles try to mimic open road conditions similar to what the vehicle encounters driving in still air. A perfect wind tunnel would also mimic these conditions if a correct ground representation and negligible blockage effects where possible to achieve. However, all full scale automotive wind tunnels suffer to some extent from blockage effects and less than ideal ground representation.

Due to the different boundary conditions between open road CFD and wind tunnel testing, a comparison of results from the two methods does not allow for distinguishing shortcomings in the numerical approach, including geometric representation of the test object, from wind tunnel interference effects. In order to be able to make such distinction, the physical test conditions, including the wind tunnel geometry, should be replicated in the simulations. This was done, for example, by Cyr et al. [1], who were able to accurately reproduce results from a $3 / 4$ open jet model scale wind tunnel by including the geometry of the test section and the boundary layer control systems (BLCS) in the CFD simulations.

\footnotetext{
† This paper was recommended for publication in revised form by Associate Editor $-$

*Corresponding author. Tel.: +46 7725045

E-mail address: emil.ljungskog@chalmers.se.

(C) KSME \& Springer 2016
}

Nayani et al. [2] simulated the flow in the high speed leg of a closed wall aeronautic wind tunnel and investigated the difference between the geometry "as designed" (construction drawings) and "as built" (point cloud from laser scanning). They found that the flow predicted for the two geometries generally agreed well, with some differences for the flow angularity near the corners of the test section. The full circuit of the same tunnel was simulated in a different work of Nayani et al. [3]. The results showed that by including the return leg with fan and corner guide vanes, some major non-uniformities known to be present in that particular configuration of the wind tunnel could be predicted. They concluded that simulating the full circuit might be useful to understand shortcomings in the flow in the circuit, but that the modeling of the fan and the anti-turbulence devices was troublesome and needed additional work.

The wind tunnel studied in this work is the Volvo Cars aerodynamic slotted wall wind tunnel (PVT), which was previously investigated numerically by Olander [4] and Wall [5]. They observed some discrepancies between the simulated and physically measured flow fields, and hypothesized that the dissemblance might be due to upstream anomalies and uncertainties in the modeling of the boundary layer control systems. One of the observed discrepancies was the longitudinal pressure distribution, which is an important test section flow quantity to match to the physical wind tunnel in order to be able to predict correct drag values in the CFD simulations. This is since even a relatively small pressure difference imposed by the tunnel between the positions of the front and rear of the tested vehicle can have a significant impact on the measured 
Fig. 1. Wind tunnel floor layout with boundary layer control systems. Adapted from [7].

drag through horizontal buoyancy effects, as noted by Waudby-Smith and Rainbird [6].

The aim of the present work is to investigate the impact of the BLCS and, to some extent, upstream anomalies on the longitudinal pressure distribution in the test section using CFD and a design of experiments approach. Instead of simulating the full circuit of the tunnel, flow angularity originating from upstream sources is introduced as an angled inflow boundary condition, and the rather complex BLCS is modeled using mass flow- and moving wall boundary conditions as described in section 3.1 .

\section{Wind tunnel}

As mentioned, the wind tunnel considered in the present work is the Volvo Car Corporation full scale aerodynamic wind tunnel (PVT) located in Gothenburg, Sweden. The physical wind tunnel and its numerical representation are discussed in the following sections.

\subsection{Physical wind tunnel}

The Volvo Cars aerodynamic wind tunnel is of closed return Göttingen-type, with a slotted wall test section of 27.06 $\mathrm{m}^{2}$ cross section area, and is equipped with a moving ground boundary layer control system as described by Sternéus et al. [7]. This system, outlined in Fig. 1, consists of a basic suction scoop, two distributed suction zones, five moving belts, and five tangential blowers. Of the five moving belts, four are wheel drive units that are placed under each wheel of the test vehicle to drive the wheel rotation during tests. The fifth belt runs under the length of the vehicle and provides the correct relative movement between the vehicle and the simulated road.

The basic suction scoop is mounted at the upstream end of the test section floor, and removes the boundary layer created along the floor of the settling chamber and contraction. The air removed by the scoop is reinjected into the wind tunnel in the plenum above the slotted test section roof. After the scoop is the first distributed suction system preventing a buildup of a new boundary layer by suction through a perforated floor with an open area ratio of $8.9 \%$. This system extends from just downstream of the scoop to the upstream edge of the turntable. A similar second distributed suction is mounted on the turntable, with an open area ratio of $4.5 \%$. Most of the air removed by the two distributed systems is reinjected into the plenum outside the slotted walls.

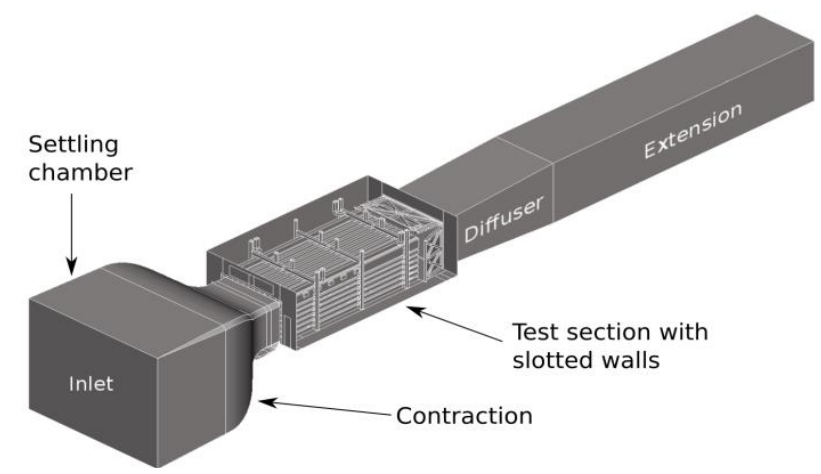

Fig. 2. Numerical geometry of PVT.

However, some of the air removed by the second distributed suction system is used by the tangential blowing devices mounted behind each of the moving belts. Each blower consists of a thin slot that blows a high speed jet of air parallel to the floor in order to refill the momentum deficit in the boundary layer building up on the static floor downstream of the belt.

\subsection{Numerical wind tunnel}

The numerical representation of the wind tunnel geometry can be seen in Fig. 2, and consists of the settling chamber, contraction, slotted walls test section, and diffuser. An extension is added aft the diffuser in order to reduce the influence from the outlet condition on the test section flow. This is the same geometry previously used by Olander [4] and Wall [5], with some minor additions of missing parts in the scoop geometry. The geometry of the test section is believed to be representative of the real tunnel. However, all details in the plenum outside of the slotted walls are not included since they are not expected to have an effect on the results of this study.

Based on the conclusions drawn by Nayani et al. [3], it is decided not to include the full circuit in the simulations. This both simplifies the complexity of the computational model and reduces the computational cost.

\section{Methodology}

The methodologies used in the present work are described in this section, beginning with the computational method, followed by a description of the design of experiments and data sampling approaches used. A description of the performed mesh resolution study is also included.

\subsection{Computational method}

Simulations are performed in STAR-CCM, using the steady-state realizable $k-\varepsilon$ turbulence model. Due to the fact that the design of experiments approach requires a substantial number of simulations, the turbulence model is chosen with respect to its relatively low computational cost. The flow field in the empty test section is expected to be suitable for a twoequation model such as realizable $k-\varepsilon$, with mostly attached flow and with no strong curvature effects. The mesh used is a trimmed cell mesh consisting of 186 million volumetric cells with 10 prism layers on the wall surfaces. The first cell height is $2 \mathrm{~mm}$, which results in $\mathrm{y}^{+}$-values of between 50 and 130 on the test section walls. The results of a mesh resolution study to ensure mesh independence are reported in section 3.4.

The inlet is specified as a mass flow inlet, with the mass flow adjusted such that the air velocity $1.2 \mathrm{~m}$ above the turn table center is $140 \mathrm{~km} / \mathrm{h}$, which is the standard speed for aerodynamic testing in the facility. This location is chosen to 


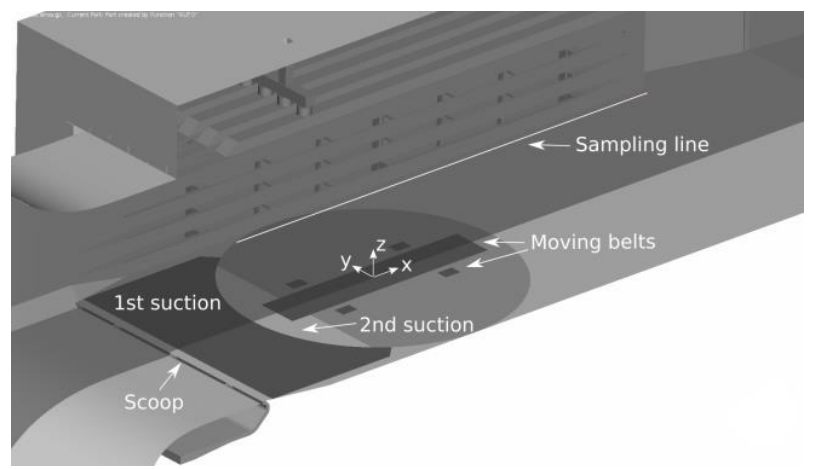

Fig. 3. Boundary layer control systems and sampling line as modeled in CFD.

coincide with the position for which the flow speed is calibrated in the physical wind tunnel. The inlet turbulence intensity is $0.1 \%$, which corresponds to the turbulence level measured in the test section during commissioning. The outlet at the end of the extension is set as an ambient pressure boundary condition.

All boundary layer control systems except for the basic scoop suction are introduced into the simulation as patches on the test section floor, with different prescribed boundary conditions. This layout can be seen in Fig. 3, which also shows the part of the scoop geometry included in the domain.

The distributed boundary layer suction systems are modeled using the approach introduced by Cyr et al. [1]. This boundary condition can be seen as a slip-wall with a wallnormal velocity component representing the mass flow removed from the domain. At each iteration, the tangential velocity components are set to the corresponding values in the first cell center. The wall normal component is fixed to a uniform value analogous to the prescribed mass flow through the surface.

The moving belts are modeled as walls with a tangential velocity specified on the surface. Due to uncertainty of the properties of the tangential blowers in the physical wind tunnel, they are omitted in the simulations. This corresponds to turning them off in a physical test.

All mass extracted by the suction systems is reinjected into the tunnel using mass flow inlets located at the reinjection positions of the physical wind tunnel.

\subsection{Design of experiments}

Design of experiments is a branch of statistics that treats quantification and explanation of variation by systematic planning and execution of experiments. This work uses a design of experiments approach to quantify the effects of the boundary conditions on the longitudinal pressure distribution in the empty test section. Six parameters, namely inlet yaw angle, inlet pitch angle, scoop mass flow, first and second distributed suction mass flow, and belt speed, are varied. These parameters are chosen since they are expected to have an impact on the flow uniformity in the test section.

The suction mass flow rates and the belt speed are allowed to vary within $\pm 20 \%$ of their baseline value, and the inlet flow angles within $\pm 1^{\circ}$, as shown in Table 1 . These variations are chosen in a range that is expected to give a measurable response, while still being reasonable with respect to the capacities of the of the corresponding systems in the physical tunnel. The baseline values for the three boundary layer suction systems are taken from the work of Wall [5], and are basically a linear interpolation where the maximum capacity of each subsystem is scaled by the ratio between the desired test section wind speed and the maximum operating wind speed of the tunnel.
Table 1. Ranges of the investigated parameters.

\begin{tabular}{lc|ccc}
\hline \hline \multicolumn{1}{c}{ Parameter } & Encoding & Low level & Baseline & High level \\
\hline Inlet yaw angle & $\mathrm{A}$ & $-1^{\circ}$ & $0^{\circ}$ & $1^{\circ}$ \\
Inlet pitch angle & $\mathrm{B}$ & $-1^{\circ}$ & $0^{\circ}$ & $1^{\circ}$ \\
Scoop mass flow & $\mathrm{C}$ & $-20 \%$ & $-23.02 \mathrm{~kg} / \mathrm{s}$ & $+20 \%$ \\
1 suction mass flow & $\mathrm{D}$ & $-20 \%$ & $-5.18 \mathrm{~kg} / \mathrm{s}$ & $+20 \%$ \\
2 suction mass flow & $\mathrm{E}$ & $-20 \%$ & $-1.38 \mathrm{~kg} / \mathrm{s}$ & $+20 \%$ \\
Belt speed & $\mathrm{F}$ & $-20 \%$ & $38.89 \mathrm{~m} / \mathrm{s}$ & $+20 \%$ \\
\hline
\end{tabular}

Since quadratic effects and interactions from the studied parameters on the longitudinal pressure distribution are expected, a Box-Behnken design [8] is employed. This is a three level quadratic design that requires 61 runs for a study of six parameters.

Construction of the design matrix and subsequent estimation of the effects is performed using the Dakota framework [9] from Sandia Labs. The effects discussed in the remainder of the paper are the polynomial coefficients $c_{i}$ and $c_{i j}$ in the fitted model

$$
\hat{f}(\mathbf{x})=c_{0}+\sum_{i=1}^{6} c_{i} x_{i}+\sum_{i=1}^{6} \sum_{j \geq i}^{6} c_{i j} x_{i} x_{j},
$$

where $\mathbf{x}$ is the parameters, and $\mathrm{c}_{0}$ corresponds to the mean response over all runs. Before the fit of equation (1) to the simulation data, each parameter is scaled to the range $[-1,1]$. This is done since the main interest of the study is to compare the relative changes of the parameters in relation to their baseline value. It shall be noted that this convention, together with the definition of the mass flows as negative values, leads to what is normally perceived as an increasing mass flow through one of the suction systems (i.e. an increased absolute value of the mass flow) is encoded as a decrease in the scaled parameter.

The factors are encoded with letters as shown in Table 1, such as A for the linear term for the inlet yaw angle and CD for the interaction effect between scoop suction mass flow and first distributed suction mass flow.

Judgment of statistical significance of the estimated effects is based on Lenth's method for analysis of unreplicated factorials [10]. This method defines a Margin of Error (ME) and a Standard Margin of error (SME) in such way that an effect can be deemed active, i.e. significant, if its absolute value is larger than SME, and can be deemed inactive if it is smaller than ME. If an effect lies in between ME and SME, the method is inconclusive on whether the effect is active or not.

\subsection{Data sampling}

The coordinate system used is a right hand system, centered at the turntable midpoint, with positive $x$ in the streamwise direction and positive $\mathrm{z}$ upwards from the floor, as indicated in Fig. 3.

The longitudinal pressure distribution is sampled on the sampling line also shown in Fig. 3. This line is located at the test section cross section center $(y=0 \mathrm{~m}, \mathrm{z}=2.05 \mathrm{~m})$, ranging from $x=-3.8 \mathrm{~m}$ to $\mathrm{x}=7.5 \mathrm{~m}$. In order to quantify the effect of each parameter on the pressure distribution, the line is divided into three zones $-3.8 \mathrm{~m} \leq \mathrm{x} \leq-0.5 \mathrm{~m},-0.5 \mathrm{~m} \leq \mathrm{x} \leq 3.5 \mathrm{~m}$ and $3.5 \mathrm{~m} \leq \mathrm{x} \leq 7.5 \mathrm{~m}$, on which the mean pressure gradient is computed from the sampled pressures. This mean value of the gradient in each zone is then used to estimate the effects by a least squares fit of the model in equation (1). The zones are chosen during the analysis such that they represent regions with clear differences between the gradients. 


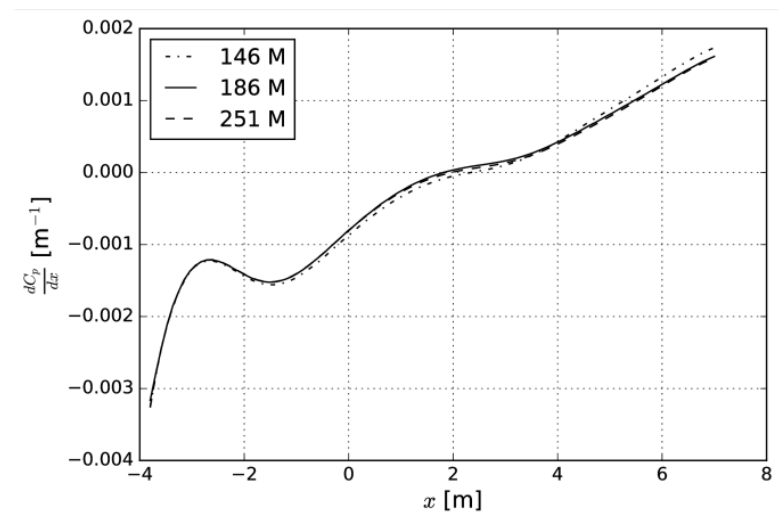

Fig. 4. Gradients for the three meshes in the mesh resolution study.

\subsection{Mesh resolution study}

In order to ensure grid independence of the simulations, a mesh resolution study is performed on three meshes of different levels of refinement, consisting of 146, 186 and 251 million cells, respectively. The coarsening and refining were done mainly at the walls, by adjusting the surface element sizes and prism layers. All three simulations were run with the baseline settings for the boundary conditions. Fig. 4 shows the pressure gradient along the test section for the three mesh resolutions. There is a very good agreement between the gradients, especially between the two finer meshes for which the gradient curves coincide. This validates the assumption of grid independence for the simulations run on the 186 million cell mesh used in the parametric study.

\section{Results and discussion}

The effects from the considered boundary conditions on the longitudinal pressure distribution in the whole test section, as well as on the pressure gradient in the three regions, are presented and discussed. This is followed by an estimation of the impact the observed changes of the longitudinal pressure distribution have on the drag measured on a vehicle in the numerical wind tunnel.

\subsection{Overall pressure distribution}

Fig. 5 shows the longitudinal static pressure coefficient distribution for all 61 cases studied. It can be seen that the general trend is similar for all runs, indicating that the inlet angularity and boundary layer control systems are not the main factors affecting the pressure distribution. This is in line with the classical theory of wind tunnel design that mainly attributes the longitudinal pressure gradient to geometrical features such as the design of the nozzle, test section walls and collector [11].

However, there are still some differences between the distributions, which are also evident when considering the gradient $d C_{P} / d x$ of the longitudinal pressure distribution in Fig. 6 . Examining these curves, it can be seen that the gradients can be divided into the three parts described in section 3.3, based on that the spread among the runs is small at the borders between the intervals. The pressure gradients in these regions are analysed in more detail in the following sections.

\subsection{Pressure gradient in the upstream region}

The upstream region, $-3.8 \mathrm{~m} \leq x \leq-0.5 \mathrm{~m}$, displays a scatter in the gradient magnitude, with no obvious tendency of the curves to form distinct groups. This lack of grouping translates

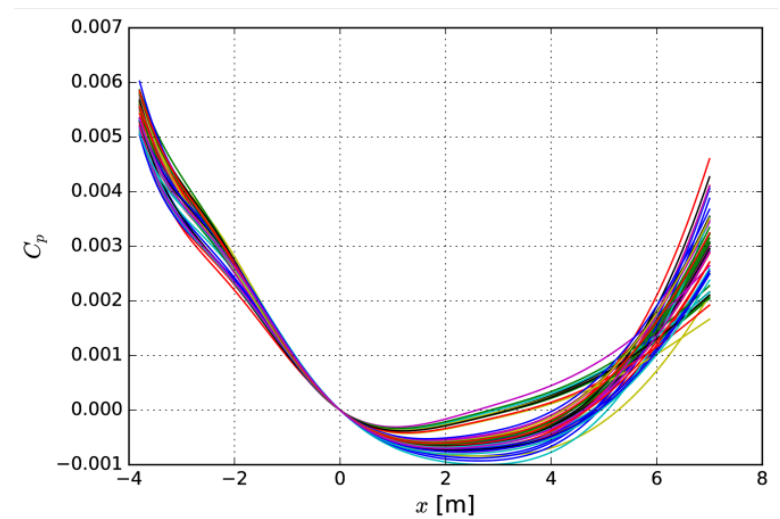

Fig. 5. Longitudinal pressure distributions for all 61 runs. All curves are shifted such that $C_{P}=0$ at $x=0$.

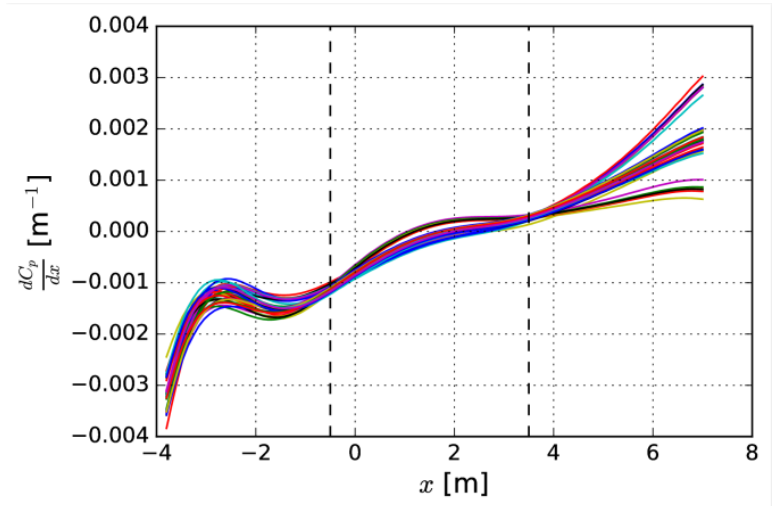

Fig. 6. Gradients for all simulations.

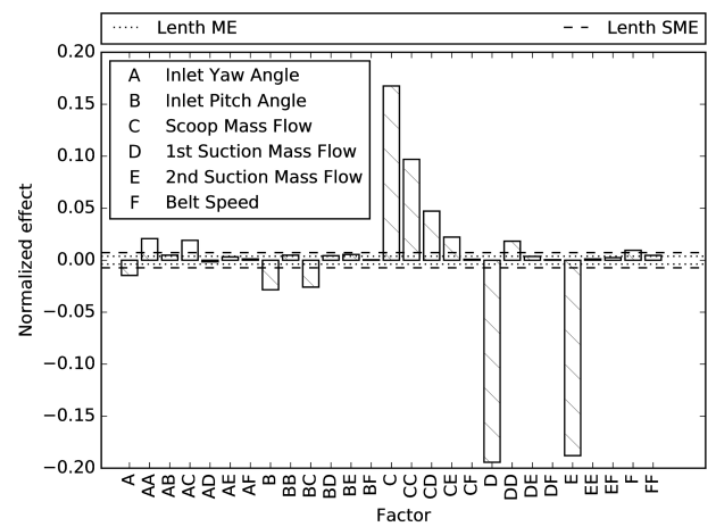

Fig. 7. Effects on mean of gradient of static pressure coefficient for $-3.8 \mathrm{~m} \leq x \leq-0.5 \mathrm{~m}$.

into the effect plot in Fig. 7 as many active effects, i.e. bars that extend beyond the dashed Lenth SME lines, of which a number are of similar magnitude. Since the Box-Behnken design is a three level design, one dominating effect would lead to the gradient curves dividing into three distinct groups in the considered region.

From Fig. 7, it can be noted that the three suction parameters $(\mathrm{C}, \mathrm{D}$ and $\mathrm{E})$ are dominating in this zone. It is expected that these systems have a relatively large impact here, since a part of the region is located above the distributed suction zones. Considering the magnitude of the effects, the basic and distributed suction seem to have comparable effects. Apart from its linear effect, the basic suction also displays a significant second-order effect (CC), and an interaction with the first distributed suction (CD). 


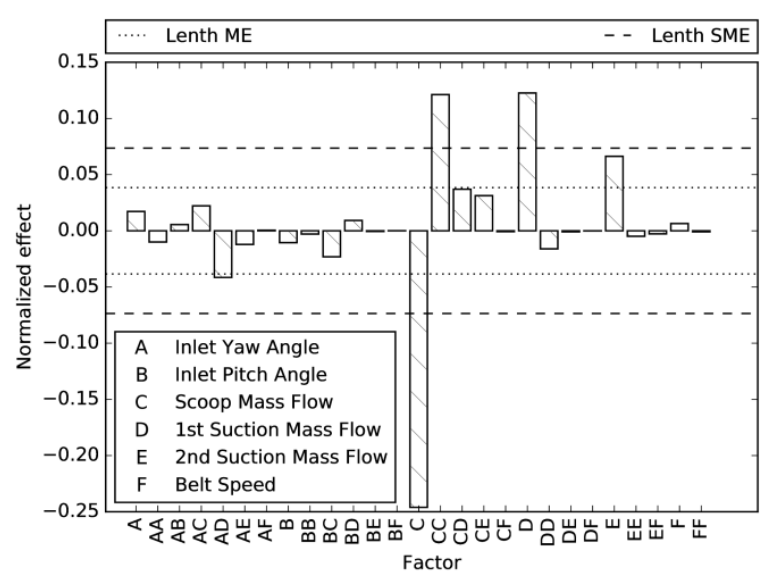

Fig. 8. Effects on mean of gradient of static pressure coefficient for $-0.5 m \leq x \leq 3.5 \mathrm{~m}$.

The effects for the two inlet angles (A and B) and some of their interactions are also significant according to Lenth's method. However, even though statistically significant, these effects are relatively small compared to the dominating ones, and are thereby not regarded as having an important impact on the pressure gradient.

\subsection{Pressure gradient in the center region}

Turning to the center region, $-0.5 m \leq x \leq 3.5 \mathrm{~m}$, it can be seen that the gradients in Fig. 6 are of relatively small magnitude. However, a tendency of bundling can be noted, which is reflected by a relatively large effect for the basic suction (C) as depicted in Fig. 8. As in the upstream region, the distributed suction systems (D and E) have a comparatively large effect on the pressure gradient, as do the second-order effect in the basic suction (CC).

\subsection{Pressure gradient in the downstream region}

The downstream region, $3.5 \mathrm{~m} \leq x \leq 7.5 \mathrm{~m}$, shows three distinct groups of the gradients in Fig. 6 . As previously mentioned, this is an indication of that one effect is dominating, which is confirmed by the high bar for the scoop suction (C) in Fig. 9. It is evident that the scoop mass flow is responsible for the main spread of the gradients, while the first distributed suction accounts for the main part of the spread within the groups. Furthermore, the magnitude of the dominating effect is much larger here compared to the two upstream regions, which is also explained by the clear bundling of the curves in Fig. 6.

As a conclusion from Fig. 7 to 9 , it can be noted that especially the linear effect in basic suction mass flow, which is dominating in all three regions, shows a tendency to switch signs between the regions. This can be explained by the gradients displayed in Fig. 6. Considering the nine curves in the group with the lowest gradient in the downstream region, it can be seen that those are the nine largest in the center zone, and reside in the low range in the upstream part. This change from low to high to low corresponds to the noted change in sign of the effects.

Fig. 10 shows the overall pressure distribution for two cases with the basic scoop suction in its low and high setting, respectively. All other factor settings are held constant between the runs. The behavior of the two curves, especially in the rear of the test section where the slope of the curves differ to a great extent, confirms the findings in Fig. 7 to 9 regarding the scoop mass flow.

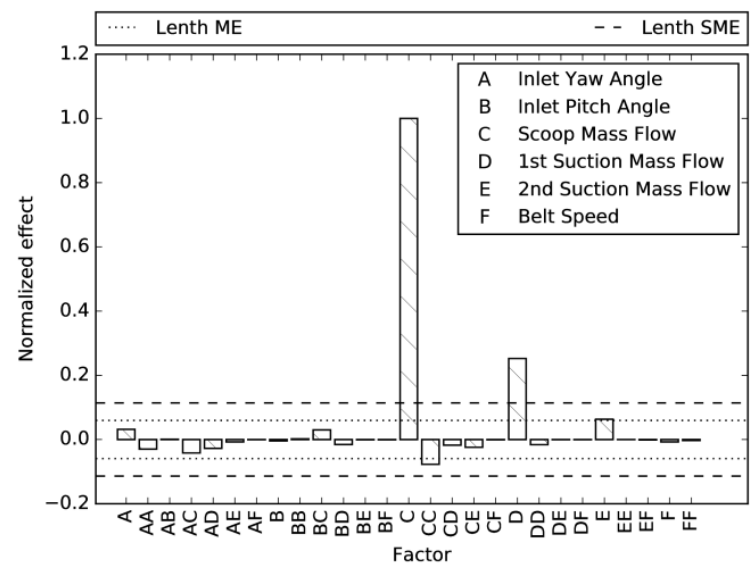

Fig. 9. Effects on mean of gradient of static pressure coefficient for $3.5 \mathrm{~m} \leq x \leq 7.5 \mathrm{~m}$.

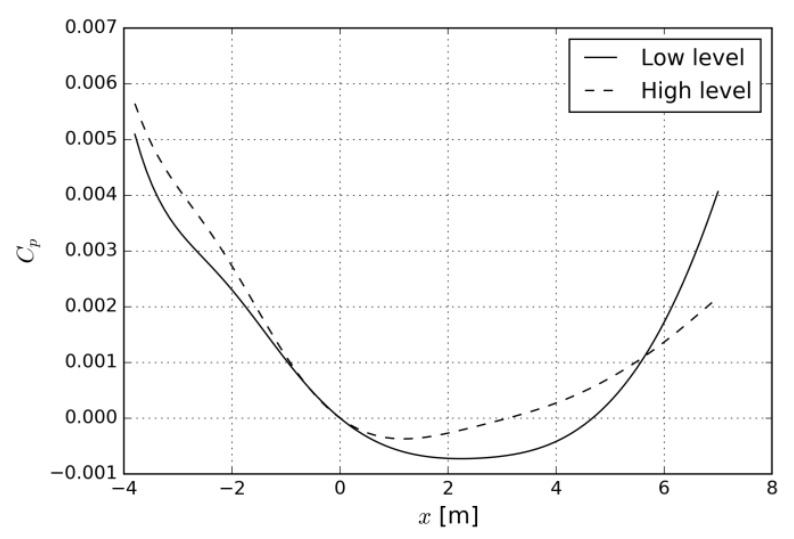

Fig. 10. Longitudinal pressure distribution for two runs with the scoop suction mass flow on low and high level.

\subsection{Estimated influence on drag measurement}

A large passenger car mounted in the test section with its front positioned at $x \approx-2.5 \mathrm{~m}$ and its rear at $x \approx 2.5 \mathrm{~m}$ is subjected to a horizontal buoyancy force whose magnitude depends on the setting of the studied boundary conditions. The pressure variation resulting in these force differences is at its maximum on the order of $\Delta \mathrm{C}_{\mathrm{P}} \approx 0.002$, according to Fig. 5, which means that the variation of the drag coefficient due to horizontal buoyancy is $\Delta C_{D} \leq 0.002$. This result is comparable to the values from physical experiments of the impact of basic suction on the measured forces on a vehicle reported by Wickern et al. [12]. Since these values are within the test-totest uncertainty of the physical wind tunnel, it is deduced that the calculated drag of a vehicle in numerical simulations of the kind performed here is fairly insensitive to the tested parameters in the ranges considered.

It shall be noted that the this work only concerns the longitudinal pressure distribution in the test section, and that other flow quantities affecting forces on the car might be influenced significantly by the tested parameters. Furthermore, since the work is based on the relatively simple $k-\varepsilon$ turbulence model, further work using more advanced simulation techniques should be performed to confirm the results. 


\section{Conclusions}

The purpose of this study has been to investigate the effects of inlet yaw angle, inlet pitch angle, basic suction scoop mass flow, first distributed suction mass flow, second distributed suction mass flow and belt speed on the longitudinal pressure distribution in the test section of the Volvo Cars aerodynamic wind tunnel using CFD. Based on the results presented, the following conclusions are drawn:

1. Within the ranges considered, the studied boundary conditions do not change the overall trend of the longitudinal pressure distribution in the empty tunnel, but can change the horizontal buoyancy experienced by a test vehicle mounted in the test section.

2. Of the parameters studied, mass flow rates through the basic and distributed suction systems show a statistically significant effect on the longitudinal pressure gradient in the empty test section.

3. The basic suction mass flow is the dominating parameter, especially when considering the regions downstream of the distributed suction zones.

4. It is estimated that the largest change in longitudinal pressure distribution between the tested settings results in a drag change of $\Delta C_{D} \leq 0.002$ on a passenger car. This is within the test-to-test uncertainty of the physical wind tunnel and is thereby not deemed as a considerable change. This should be confirmed in future work using more advanced simulation methods.

\section{Acknowledgements}

This work was funded by FFI (Fordonsstrategisk Forskning och Innovation, Strategic Vehicle Research and Innovation) through Vinnova (Sweden's innovation agency) and Volvo Cars.

The simulations were performed on resources provided by the Swedish National Infrastructure for Computing (SNIC) at PDC Centre for High Performance Computing (PDC-HPC)

\section{References}

[1] S. Cyr, K.-D. Ih, and S.-H. Park. "Accurate Reproduction of Wind-Tunnel Results with CFD”. SAE Technical Paper 2011-01-0158. Apr. 12, 2011. doi: 10.4271/2011-01-0158.

[2] S. Nayani, W. L. Sellers, S. E. Brynildsen, and J. L. Everhart. "Numerical Study of the High-Speed Leg of a Wind Tunnel". AIAA Science and Technology Forum and Exposition. Kissimmee, FL, United States, Jan. 5, 2015.

[3] S. Nayani, W. L. Sellers, A. F. Tinetti, S. E.Brynildsen, and E. L. Walker. "Numerical Simulation of a Complete Low-Speed Wind Tunnel Circuit". 54th AIAA Aerospace Sciences Meeting. American Institute of Aeronautics and Astronautics.

[4] M. Olander. "CFD Simulation of the Volvo Cars Slotted Walls Wind Tunnel". Masters Thesis. Chalmers University of Technology, 2011.

[5] A. Wall. "Simulating the Volvo Cars Aerodynamic Wind Tunnel with CFD". Masters Thesis. Chalmers University of Technology, 2013.

[6] P. M. Waudby-Smith and W. J. Rainbird. "Some Principles of Automotive Aerodynamic Testing in Wind Tunnels with Examples from Slotted Wall Test Section Facilities". SAE Technical Paper 850284. Feb. 1, 1985. doi: 10.4271/850284.

[7] J. Sternéus, T. Walker, and T. Bender. "Upgrade of the Volvo Cars Aerodynamic Wind Tunnel". SAE Technical Paper 2007-01-1043. Apr. 16, 2007. doi: 10.4271/2007-01-1043.

[8] G. E. P. Box and D. W. Behnken. "Some New Three Level Designs for the Study of Quantitative Variables". Technometrics 2.4 (Nov. 1, 1960), pp. 455475. doi: 10.1080/00401706.1960.10489912.

[9] B. M. Adams et al. "Dakota, A Multilevel Parallel Object-Oriented Framework for Design Optimization, Parameter Estimation, Uncertainty Quantification, and Sensitivity Analysis: Version 6.0 User's Manual". Sandia Technical Report SAND2014-4633 (July 6, 2014).

[10] R. V. Lenth. "Quick and Easy Analysis of Unreplicated Factorials". Technometrics 31.4 (1989), pp. 469-473. doi: 10.2307/1269997.

[11] E. Mercker and J. Wiedemann. "On the Correction of Interference Effects in Open Jet Wind Tunnels". SAE Technical Paper 960671. Feb. 1, 1996. doi: 10.4271/960671

[12] G. Wickern, S. Dietz, and L. Luehrmann. "Gradient Effects on Drag Due to Boundary-Layer Suction in Automotive Wind Tunnels". SAE Technical Paper 2003-01-0655. Mar. 3, 2003. doi: 10.4271/2003-010655 . 